\title{
Therapeutic Heroin in the XXI Century
}

Authors: B. Martins, A. Matos \& M. Sardinha (Unidade Local de Saúde do Norte Alentejano, EPE)

\section{Background and aims}

Europe has experienced different waves of heroin addiction. In 2015, the average prevalence of high-risk opioid use among adults was estimated at $0.4 \%$ of the European Union population (i.e., 1.3 million opioid users). Nowadays, many of the ageing high-risk opioid users are very likely to have been in contact with substitution treatment services for over two decades (EMCDDA, 2017).

In order to compare with opioid agonists treatment programs, our goal is to review what benefits therapeutic heroin programs may have, in spite of social and political resistance.

\section{Materials and methods}

A research in Medline was done for the following terms: therapeutic heroin and therapeutic diamorphine. Only the relevant articles, published in English and French, were selected.

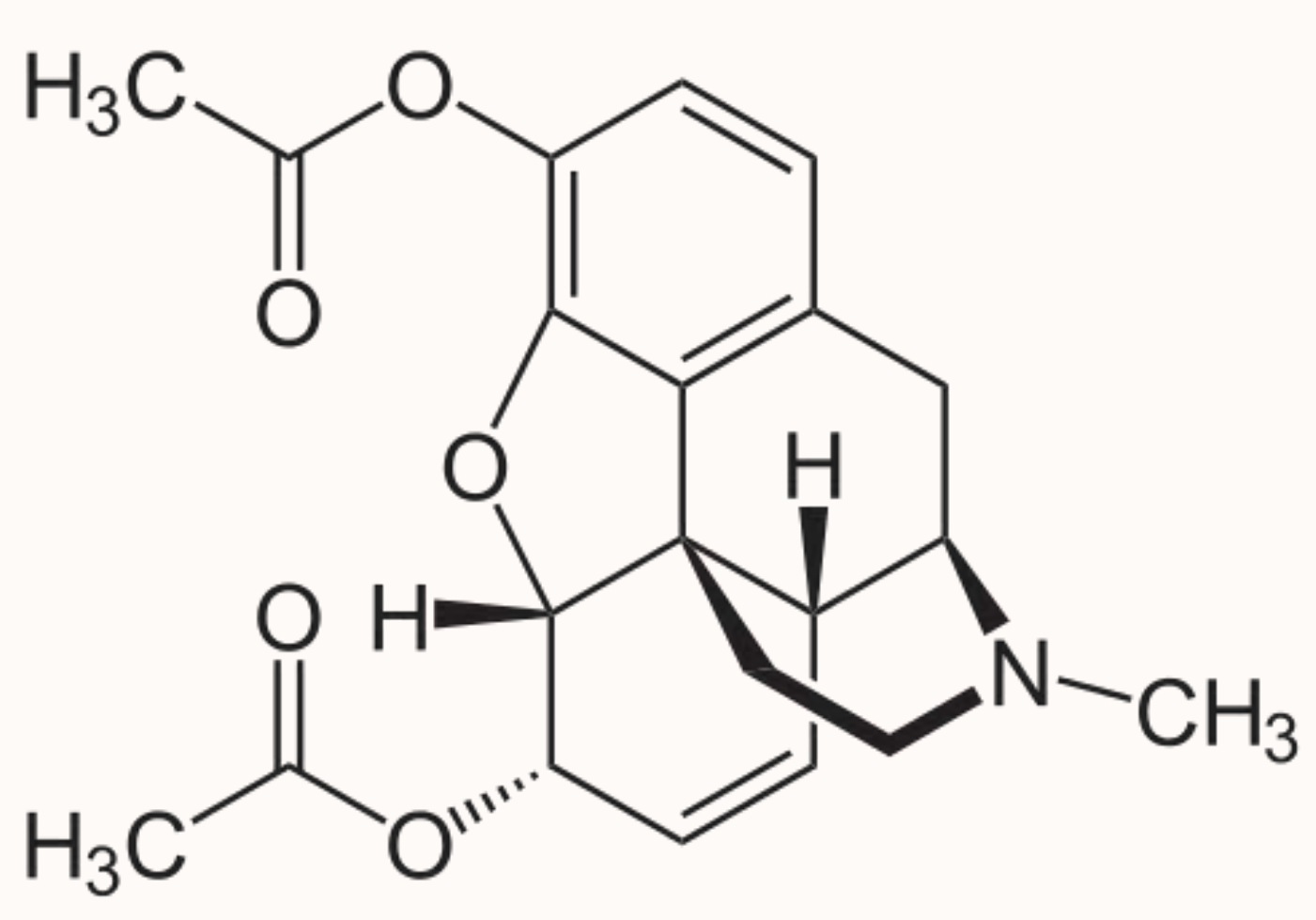

\section{Heroin or Diamorphine}

It is a semi-synthetic drug derived from morphine, whose effects are dose-related and related to the rate of absorption.

When taken under the controlled clinical conditions, it is not neurotoxic and has few long-term side effects beside constipation and dependence.

It reaches the brain rapidly, where it is deacetylated into 6monoacetylmorphine and then into morphine, which binds to $\mathrm{m}$ opioid receptors to produce the drug's euphoric, analgesic, and anxiolytic effects.

\section{Results}

Heroin use faces unquestionable risks, such as the high mortality rate (1 to $3 \%)$ or the high incidence of criminal behavior among heroin users (Demaret, et al., 2013).

Yet, a meta-analysis of six randomized clinical trials (comparing the effectiveness of supervised injectable heroin to oral methadone) concluded that therapeutic heroin was more effective than methadone in suppressing street heroin use, among people who had failed to respond to substitution treatment (Bell, et al., 2017).

Users under heroin-assisted treatment are provided with a form of pharmaceutical-grade heroin injection solution. The medical supervision refrains from drastic changes in dose and provide post-injection monitoring, which minimizes the occurrence of overdoses. Thus, patients in heroin-assisted treatment are relieved from the major complex of problems that defines illicit heroin use (Fischer, et al., 2007).

\section{Conclusions}

Although the present paradigm of heroin dependence treatment is mostly associated with risk minimizing programs and with opioid agonists, psychiatrists and political leaders should be aware that other pharmacological options could be considered in order to maximize the success of heroin use disorder treatment. So far, only a few European countries (namely Switzerland and Netherlands) have implemented Heroin-Assisted Treatments.

An integrative approach, including psychosocial intervention, remains however imperative. 